\title{
An improved reconstruction method for the AMIGA detectors
}

\author{
Juan Manuel Figueira ${ }^{* a}$ for the Pierre Auger Collaboration ${ }^{b}$ \\ anstituto de Tecnologías en Detección y Astropartículas (CNEA, CONICET, UNSAM), \\ Centro Atómico Constituyentes, Comisión Nacional de Energía Atómica, \\ Av. General Paz 1499 (B1650KNA) San Martín, Buenos Aires, Argentina. \\ ${ }^{b}$ Observatorio Pierre Auger, Av. San Martín Norte 304, 5613 Malargüe, Argentina. \\ E-mail: auger_spokespersons@fnal.gov \\ Full author list: http://www.auger.org/archive/authors_icrc_2017.html
}

\begin{abstract}
The Auger Muon and Infill Ground Array (AMIGA) is a muon detector that is currently being built as part of AugerPrime, the upgrade of the Pierre Auger Observatory. It consists of $30 \mathrm{~m}^{2}$ plastic scintillator counters buried $2.3 \mathrm{~m}$ underground and water-Cherenkov detectors at the surface, organized in a periodic $750 \mathrm{~m}$ triangular array, and deployed over an area of $23.5 \mathrm{~km}^{2}$. Each counter is composed of three $10 \mathrm{~m}^{2}$ modules segmented into 64 scintillator strips. Two positions of the engineering array were further equipped with "twin" detectors to assess reconstruction uncertainties. AMIGA allows for direct measurement of the muon content of air showers with primary energies above $10^{17} \mathrm{eV}$. In this work, the detector reconstruction strategy is revisited and the bias induced by particles traversing two adjacent strips, the so-called corner-clipping effect, is thoroughly analyzed. A bias correction based on end-to-end simulations of both the air showers and the detector response is presented. The improved reconstruction method was applied to experimental data acquired by the AMIGA engineering array, and preliminary results of the muon content estimator $\rho_{450}$ (muon density $450 \mathrm{~m}$ from the shower axis) are presented.
\end{abstract}

35th International Cosmic Ray Conference - ICRC2017

10-20 July, 2017

Bexco, Busan, Korea

* Speaker. 


\section{Introduction}

The Pierre Auger Observatory [1] is a hybrid detector, composed of 27 air-fluorescence telescopes (the fluorescence detector, FD) that have a view of the atmosphere over an arrangement of 1660 water-Cherenkov particle detector stations spread over $3000 \mathrm{~km}^{2}$ (the surface detector, SD). The SD stations are situated on a triangular grid with a spacing of $1500 \mathrm{~m}$ (mostly, with some closer together as described below), and the FD telescopes are located at four sites at the edge of the SD array pointing inwards.

The Observatory is embarking on its next phase, named AugerPrime. As one of the enhancements to the detection system, a dedicated detector to directly measure the muon content of air showers, AMIGA [1, 2, 3], is being built. AMIGA is a joint system of 61 water-Cherenkov stations and buried plastic scintillation counters arranged in a $750 \mathrm{~m}$ spacing array nested inside the $1500 \mathrm{~m}$ array, that extends over an area of $23.5 \mathrm{~km}^{2}$. The $750 \mathrm{~m}$ array is fully efficient from $3 \times 10^{17} \mathrm{eV}$ onwards for air showers with zenith angles $\leq 55^{\circ}[4]$. The buried scintillators (the underground muon detector, UMD) are the core of the detection system for the muonic component of air showers since the electromagnetic component is largely absorbed by the overburden. To effectively shield the UMD, scintillators are situated $2.3 \mathrm{~m}$ underground, which corresponds to $\sim 540 \mathrm{~g} / \mathrm{cm}^{2}$ of vertical mass from the local soil and imposes a cutoff for vertical muons of about $1 \mathrm{GeV}$. The scintillator plane of each UMD station is highly segmented, composed of three $10 \mathrm{~m}^{2}$ modules comprising sixty four $400 \mathrm{~cm} \times 4.1 \mathrm{~cm} \times 1.0 \mathrm{~cm}$ strips each or, in the case of the engineering array, composed of two $5 \mathrm{~m}^{2}$ modules made up of sixty four $200 \mathrm{~cm} \times 4.1 \mathrm{~cm} \times 1.0 \mathrm{~cm}$ strips each and two $10 \mathrm{~m}^{2}$ modules. The SD $750 \mathrm{~m}$ array was completed in September 2011 while the UMD engineering array, consisting of seven stations arranged in a hexagonal layout (vertices and center), has been operational since February 2015.

When a muon crosses a UMD scintillator strip a fraction of the produced light reaches a photomultipler tube (PMT), which converts a bunch of photons into a current pulse. The pulse is amplified and inverted to produce the so-called analog trace, which is typically $\lesssim 30 \mathrm{~ns}$ wide. Then a pulse amplitude discriminator produces a constant output if the input signal is higher than a programmable threshold voltage set at $\sim 30 \%$ of the average single photoelectron amplitude. Finally, the pulse is sampled at $320 \mathrm{MHz}$ by a field programmable gate array (FPGA). Samples can be either a logical "1" or "0" depending on whether the incoming signal was above or below the discrimination threshold. So, at the end every channel has a digital trace consisting of a binary string. UMD scintillator modules receive the trigger signal from their associated SD station. The lowest level trigger (T1) of the surface detectors is used. Once a T1 condition is fulfilled, its UMD companion freezes a $6.4 \mu$ s data sample into a local buffer capable of storing 1024 triggers.

\section{Main sources of counting biases}

The main sources of counting biases in UMD modules with PMTs are: i) crosstalk, ii) muon pile-up, and iii) corner-clipping muons. Crosstalk and corner-clipping muons tend to an overcounting of the real number of muons impinging the counter while, on the other hand, muon pile-up leads to undercounting. Crosstalk and muon pile-up were already considered in the previous reconstruction counting strategy. We will present here a new reconstruction method that also takes into account the corner-clipping effect. In the following subsections, the three sources of counting biases will be briefly explained. 


\subsection{Crosstalk}

Normally, in a multianode PMT (e.g., the Hamamatsu UBA H8804-200MOD [5], as used in the engineering array of the UMD), the photoelectrons emitted from the photocathode are accelerated and guided by a focusing mesh towards the first dynode of a pixel where secondary electrons are released; these electrons are multiplied by a factor of $\sim 10^{6}$ in a cascade process from the first to the last dynode of the same pixel, before finally reaching an anode connected to an output processing circuit. On occasion, the electronic crosstalk phenomenon may occur, which consists of a secondary electron leaking out from the cascade multiplication process of the original pixel into a dynode of an adjacent pixel and triggering another cascade multiplication process on it. In such a situation, the anode connected to the adjacent pixel would have an output current pulse similar to the one produced by a single photoelectron emitted from the photocathode. There is also a chance for the optical crosstalk phenomenon to occur, which happens when a photon (out of a group of them) arriving at the PMT from the fiber connected to a certain pixel strikes the photocathode near the focusing electrodes of a neighboring pixel, mainly due to the numerical aperture of the fiber or a small misalignment of the fiber with the pixel.

\subsection{Pile-up}

Muon traces typically span $\lesssim 30 \mathrm{~ns}$ and, due to exponential decay processes involved in the light emission of the scintillators and fibers, can have a complex time structure. Because of this, after the pulse discrimination, a digital muon trace may not comprise only a sequence of consecutive positive samples ("1s"), but could also have null samples ("0s") in between. For this reason, to prevent the binary string produced by one muon to be counted as two or more, an inhibition time window, over which the searching process for a muon identification pattern is stopped, has to be applied starting from the first identified positive sample on the binary string. As a consequence of the amplitude discrimination, once this inhibition time window is triggered by a muon arriving at the scintillator strip, it is not possible to identify whether another muon arrives at the same strip over the same time interval spanned by the window. Therefore, the so-called muon pile-up effect, which can be defined as two or more muons arriving at the same detection strip over a time interval shorter than an inhibition time window, is hampering the muon counting, among other factors. Even though this phenomenon was taken into account in the design of the muon counter by choosing a high segmentation (64 scintillation strips per module), it can still be encountered in situations such as counters sufficiently close to the shower core or counters in high-energy events.

\subsection{Corner clipping}

The arrangement of the scintillator strips in the UMD counters [2] is such that most of the muons impinging the detector will pass through only one strip, i.e., they will go from the top face to the base of a strip without crossing any other. Some muons, however, due to their directions and impact point, will cross a lateral face of a scintillator strip. This is the so-called corner-clipping effect. In this situation, since the strips are touching each other along their lateral faces, it is most likely that the muon will go across two strips. In such a case, the muon could be detected in both strips, only in one of them, or not at all, depending on whether the deposited energy on each strip is above or below the threshold required to be reconstructed. Additionally, while they are traveling along the surrounding area of the counter, muons will scatter knock-on electrons at very forward angles which may have enough energy to produce secondary ionization. In case they are produced, 
it is most likely that they will be injected in the same strip as the muon, but it is also possible that some of them are injected into an adjacent strip, depositing enough energy to be detected and, therefore, contributing as another overcounting source. Figure 1 illustrates simulation results of the mean relative counting bias (i.e., the relative deviation of the measured value from the expected value) caused by corner-clipping as a function of the distance to the shower axis, averaged over azimuth angle. As expected for geometrical reasons, the bias increases with zenith angle due to the increased number of corner-clipping muons. For zenith angles of $0^{\circ}$ and $45^{\circ}$, the mean overcounting becomes $\sim 5 \%$ and $\sim 20 \%$ respectively, and is almost independent of the distance to the shower axis or the primary energy.

\section{The improved reconstruction method}

The reconstruction begins with a searching process for a recognition pattern on the digital trace of every counter channel. This pattern, which was studied in Ref. [6], must discriminate between digital traces produced by muons and digital traces from single photoelectron-like pulses produced by crosstalk and, eventually, thermal fluctuations. Since single photoelectron pulses have a typical width of $\sim 4 \mathrm{~ns}$, and the digital sampling is performed every $3.125 \mathrm{~ns}$, the possible digital traces that a single photoelectron can produce comprise null samples (000), an isolated positive sample (010) or two consecutive and isolated positive samples (0110). The used recog-

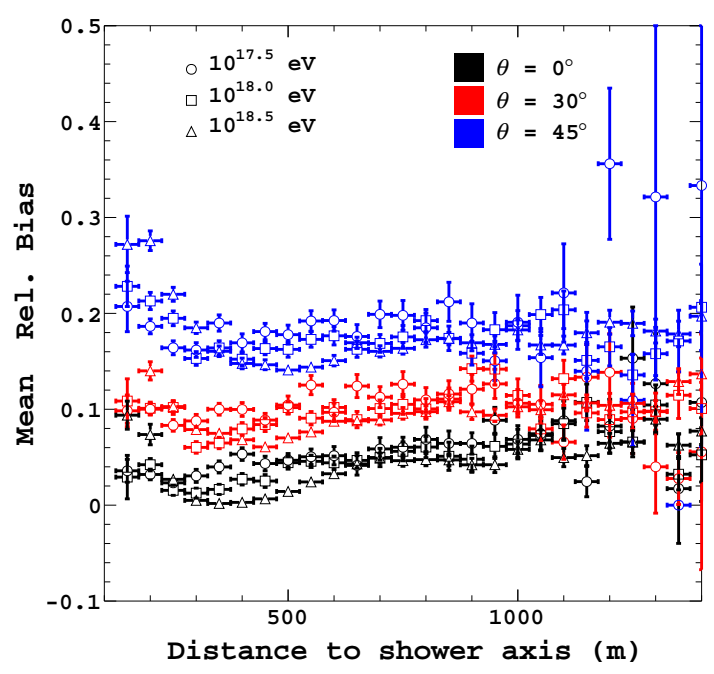

Figure 1: Simulations of the biases due to the corner-clipping effect vs. the distance to the shower axis for showers of different zenith angles and energies. nition pattern for muon detection demands, therefore, an extra sample between two positive samples, irrespective of whether it is positive or null. Thus, all the traces which contain at least the strings 101 or 111 will be considered as signals generated by at least one muon while those produced by single photoelectron-like pulses from crosstalk will not be taken into account. Once the recognition pattern of a muon is found in a counter channel, an inhibition time window is applied from the detection of the first positive sample in the digital trace. The length of the inhibition time window was also studied in Ref. [6] from the widths at the discrimination level of muon pulses measured in the laboratory, and it was found that it should be equal to or larger than $30 \mathrm{~ns}$ to prevent any double counting due to the pulse structure of a single muon, as explained above.

Once the 64 digital traces of a UMD module are examined over the $6.4 \mu$ s that the event acquisition lasts to find patterns generated by muons, the start times of the muon signals are determined as those corresponding to the first positive samples of the patterns. To perform the muon pile-up correction, the event is divided in time windows with the same duration as the inhibition time window. Subsequently, the number of channels with signal $N_{\mathrm{s}}$ is determined in each window. Then, as shown in Ref. [7], the bias due to muon pile-up can be corrected by means of the unbiased statistical correction

$$
N_{\mathrm{s}, \mathrm{cor}}=-N_{\mathrm{seg}} \ln \left(1-N_{\mathrm{s}} / N_{\mathrm{seg}}\right)
$$



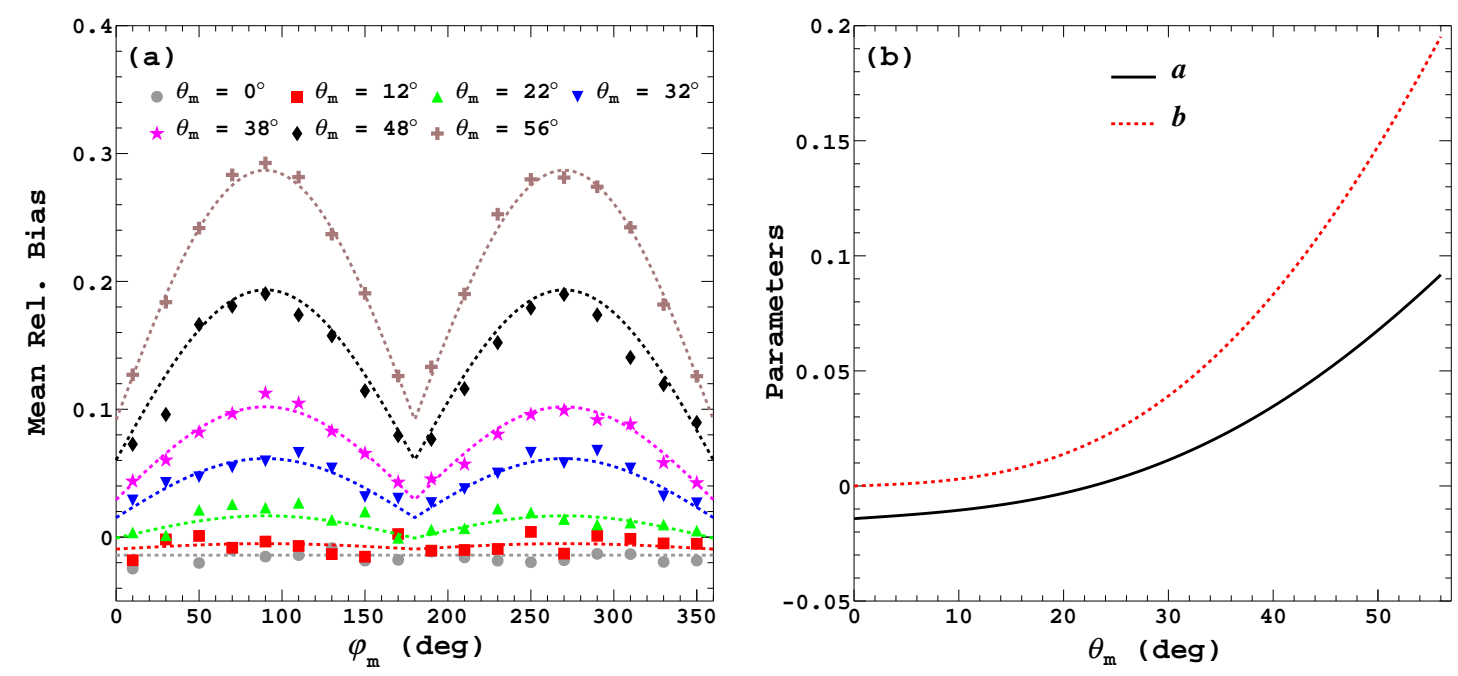

Figure 2: (a) Simulation results of the dependence of the bias on $\varphi_{\mathrm{m}}$ and $\theta_{\mathrm{m}}$, averaged over proton- and iron-induced showers of $10^{18.0} \mathrm{eV}$ and $10^{18.5} \mathrm{eV}$, with $\varphi_{\mathrm{m}}$ grouped into $20^{\circ}$ wide bins (symbols), and the corresponding curves obtained with the parametrization of Eq. 3.2 that best fits the data globally (lines). (b) Resulting coefficients $a(\theta)$ and $b(\theta)$ of the parametrization.

where $N_{\mathrm{s}, \mathrm{cor}}$ is the number of corrected signals and $N_{\mathrm{seg}}=64$ is the segmentation of a module.

To address the problem that arises due to the bias of the corner-clipping effect, a geometrical correction based on simulations was developed. This reconstruction bias was determined on a module-by-module basis by examining the quantity $B=\left(N_{\mathrm{s}, \mathrm{cor}}-N_{\mu \text {,inj }}\right) / N_{\mu \text {,inj, }}$, i.e., the relative difference between the number of reconstructed signals in the 64 strips of the UMD module $\left(N_{\mathrm{s}, \mathrm{cor}}\right)$ and the number of muons impinging on the module $\left(N_{\mu, \text { inj }}\right)$. The simulation library employed to develop the geometrical correction was composed of proton- and iron-induced air showers of energies $E=10^{18.0} \mathrm{eV}$ and $10^{18.5} \mathrm{eV}$, zenith angles $\theta=0^{\circ}, 12^{\circ}, 22^{\circ}, 32^{\circ}, 38^{\circ}, 48^{\circ}$ and $56^{\circ}$, respectively, azimuth angles uniformly distributed in the range $0^{\circ}$ to $360^{\circ}$, and with QGSJET-II-04 as the high-energy hadronic interaction model [8]. The zenith and azimuth angles of the shower axis, $\theta$ and $\varphi$, respectively, were obtained by means of the SD reconstruction. Then, taking into account the azimuth rotation of each UMD module with respect to the SD coordinate system, $\varphi_{0}$, the zenith and azimuth angles of the shower axis were referenced to the coordinate system of each module by using $\theta_{\mathrm{m}}=\theta, \varphi_{\mathrm{m}}=\varphi+\varphi_{0}$. Relative to the module coordinate system, the projection of the shower axis into the $x-y$ plane (in which the module itself is located) is parallel to the longitudinal direction of the strips when $\varphi_{\mathrm{m}}=0^{\circ}$ or $180^{\circ}$, and perpendicular to them when $\varphi_{\mathrm{m}}=90^{\circ}$ or $270^{\circ}$. Figure 2(a) shows the dependence of the relative bias correction on $\varphi_{\mathrm{m}}$ and $\theta_{\mathrm{m}}$, averaged over proton- and iron-induced showers of $10^{18.0} \mathrm{eV}$ and $10^{18.5} \mathrm{eV}$, with $\varphi_{\mathrm{m}}$ grouped into $20^{\circ}$ wide bins. Since for $\theta_{\mathrm{m}} \neq 0$ the corner-clipping effect reaches minimum and maximum values at $\varphi_{\mathrm{m}}=0^{\circ}$ and $180^{\circ}$, and $\varphi_{\mathrm{m}}=90^{\circ}$ and $270^{\circ}$, respectively, the bias correction was parametrized as

$$
C_{\text {bias }}\left(\theta_{\mathrm{m}}, \varphi_{\mathrm{m}}\right)=a\left(\theta_{\mathrm{m}}\right)+b\left(\theta_{\mathrm{m}}\right)\left|\sin \left(\varphi_{\mathrm{m}}\right)\right|,
$$

where $a\left(\theta_{\mathrm{m}}\right)=x_{0}+x_{1}\left(1+x_{2} \cos \left(\theta_{\mathrm{m}}\right)\right) \sin \left(\theta_{\mathrm{m}}\right), b\left(\theta_{\mathrm{m}}\right)=x_{3}\left(1+x_{4} \cos \left(\theta_{\mathrm{m}}\right)\right) \sin \left(\theta_{\mathrm{m}}\right)$ and $x_{i}$ (for $0 \leq i \leq 4)$ are free parameters. A global least-squares fitting was carried out by minimizing $R^{2}=\sum_{k=1}^{N}\left(B_{k}-C_{\text {bias }}\left(\theta_{\mathrm{m}, k}, \varphi_{\mathrm{m}, k}, x_{0}, x_{1}, x_{2}, x_{3}, x_{4}\right)\right)^{2}$, where the sum was performed over the total 

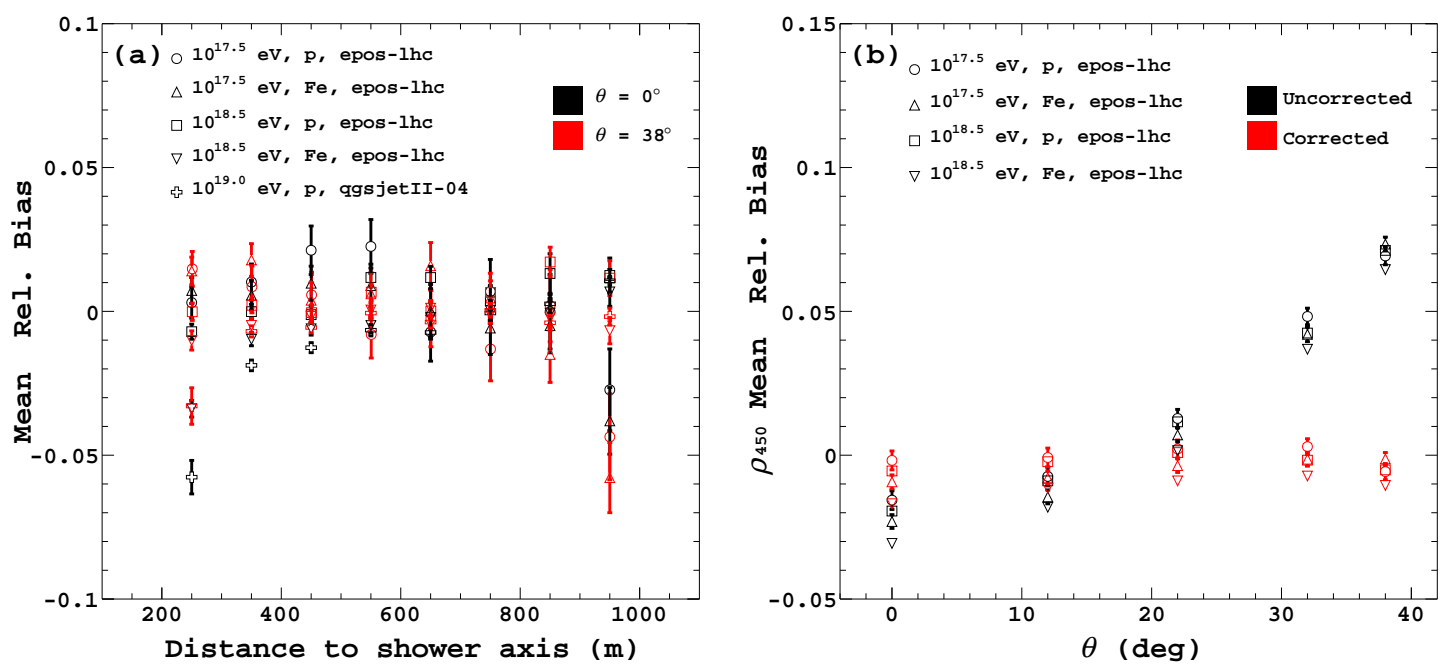

Figure 3: Simulation results of the counting biases obtained with the improved reconstruction that performs the correction due to the corner-clipping effect, for (a) the number of muons at station level vs. distance to the shower axis, and (b) the shower size $\left(\rho_{450}\right)$ vs. zenith angle.

number of modules $N$ available in the whole simulated data. As result, the best-fitting parameters are $x_{0}=-0.014 \pm 0.002, x_{1}=0.27 \pm 0.02, x_{2}=-0.94 \pm 0.04, x_{3}=0.52 \pm 0.03$, and $x_{4}=$ $-0.98 \pm 0.02$. The values taken by $a$ and $b$ as a function of $\theta_{\mathrm{m}}$ are displayed in Fig. 2(b), and the good agreement between the parametrization and the simulated data can be seen in Fig. 2(a). The improved reconstruction obtained in this work consists of applying a correction factor given by $f(\theta, \varphi)=\left(1+a(\theta)+b(\theta)\left|\sin \left(\varphi+\varphi_{0}\right)\right|\right)^{-1}$ on a module-by-module basis.

For testing this approach, independent sets of proton- and iron-induced showers were simulated by using the CORSIKA software package [9]. The energies and zenith angles were fixed to $E=10^{17.5} \mathrm{eV}, 10^{18.5} \mathrm{eV}$ and $10^{19.0} \mathrm{eV}$, and $\theta=0^{\circ}, 12^{\circ}, 22^{\circ}, 32^{\circ}$ and $38^{\circ}$, respectively, while the azimuth angles were generated randomly in the range $0^{\circ}$ to $360^{\circ}$ for each air shower. QGSJET-II-04 and EPOS-LHC [10] were chosen as the high-energy hadronic interaction models for $E=10^{19.0} \mathrm{eV}$, and $E=10^{17.5} \mathrm{eV}$ and $10^{18.5} \mathrm{eV}$, respectively. The simulation of the detector and the reconstruction method were carried out by using the Offline framework [11], and for each energy and zenith angle, 120 simulations/reconstructions were performed. Figures 3 (a) and (b) show the counting biases with the improved reconstruction. While with the former reconstruction method the biases are $\sim 5 \%$ and $\sim 10 \%$ for $\theta=0^{\circ}$ and $38^{\circ}$, respectively, once the developed correction is applied, the large bias vanishes for $\theta=38^{\circ}$ and remains negligible for $\theta=0^{\circ}$, as can be seen in Fig. 3(a). The bias correction works well over the whole range of distances to the shower axis and for all considered zenith angles, primaries, models, and energies. The azimuthal dependence is also removed. Figure 3(b) shows the biases of the density of muons at $450 \mathrm{~m}$ from the shower axis $\left(\rho_{450}\right)$ before and after applying the geometrical correction. The values of the muon content $\rho_{450}$ are obtained by fitting a KASCADE-Grande-like function as expressed in Ref. [12] to the lateral distribution functions of muon densities. As can be seen, the biases from uncorrected muon numbers at the station level obtained with the former reconstruction increase with $\theta$, taking a value of $\sim 8 \%$ for $\theta=38^{\circ}$. On the other hand, corrected muon numbers at the station level yield biases in $\rho_{450}$ values almost independent of $\theta$ and contained in a $\pm 2 \%$ range. 


\section{Muon content at $450 \mathrm{~m}$ vs. primary energy}

In this section the previously described improved reconstruction method is applied to data acquired with the UMD engineering array, and the energy dependence of the muon density at $450 \mathrm{~m}$ from the shower axis is presented. The data set comprises all cosmic-ray events collected from October 21st 2015 to October 15th 2016. In addition to the described bias correction, inefficiencies of the $5 \mathrm{~m}^{2}$ and $10 \mathrm{~m}^{2}$ modules due to light attenuation in the fibers were corrected for by dividing the reconstructed muon densities by 0.91 and 0.83 , respectively, values obtained from laboratory measurements. The SD provided the energy and arrival direction of the pri-

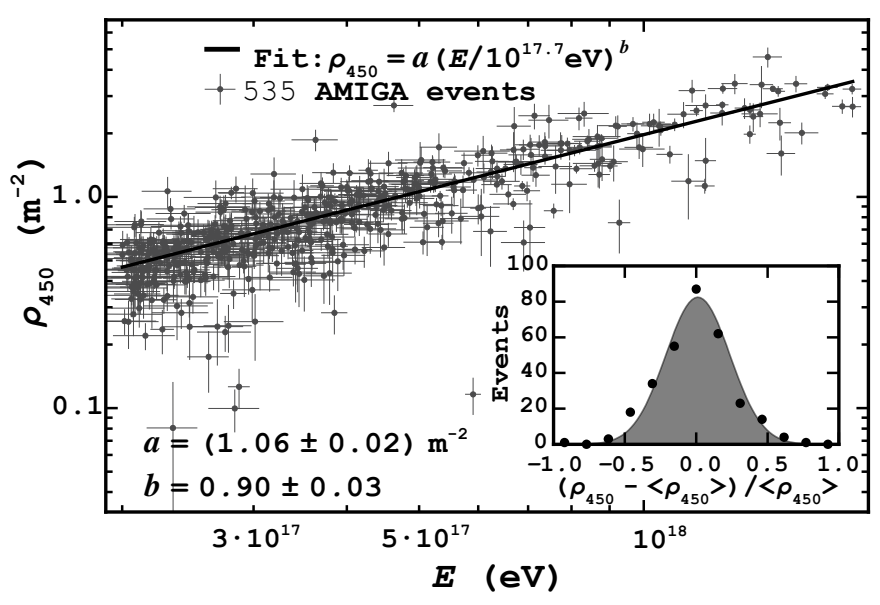

Figure 4: Dependence on the shower energy $E$ of the muon content $\rho_{450}$ for 535 selected events acquired by the AMIGA engineering array above $10^{17.3} \mathrm{eV}$ (gray dots) and a fit to the data by the power law given in Eq. 4.1 (line). The error bars display statistical detection uncertainties only. The inset shows the distribution of the deviations of the data points from the fitted curve.

mary cosmic rays from the timing and amplitudes of the signals produced by the air-shower particles in the stations [1]. The standard SD reconstruction algorithm and trigger condition were applied. The previously determined lateral trigger probability (LTP) of measuring 1 muon in $10 \mathrm{~m}^{2}$ as a function of the radial distance and primary energy was used to select high-quality events. For each event, the radial distance for an LTP $\geq 0.7$ was calculated. Within this radial range the number of modules $N_{\mathrm{m}}$ and their maximal spacing $d_{\max }$ were determined. Then, to ensure a sufficiently spaced number of counters, the event was selected if: $N_{\mathrm{m}}>1$ and $d_{\max }>250 \mathrm{~m}$, or $N_{\mathrm{m}}>2$ and $d_{\max }>165 \mathrm{~m}$, or $N_{\mathrm{m}}>3$ and $d_{\max }>125 \mathrm{~m}$. Zenith angles up to $30^{\circ}$ and energies higher than $10^{17.3} \mathrm{eV}$ were also required. Above this energy threshold, the trigger efficiency of the SD infill is $\geq 90 \%$. After requiring those conditions, 535 events remained. UMD stations closer to the shower axis than $200 \mathrm{~m}$ were not taken into account.

$\rho_{450}$ was used as an estimator of the muon content since its mean value is a robust composition estimator [13]. Because it is expected that the dependence of the muon content on the energy follows a power law [13], the experimental data shown in Fig. 4 was fitted by the expression

$$
\rho_{450}=a\left(E / 10^{17.7} \mathrm{eV}\right)^{b},
$$

where the fitting parameters $a$ and $b$ represent the average muon content at $10^{17.7} \mathrm{eV}$ and the logarithmic gain of muons with growing energy, respectively. As can be seen, the fitted model is in good agreement with data. It was found that $a=(1.06 \pm 0.02) \mathrm{m}^{-2}$ and $b=0.90 \pm 0.03$, the latter being in accord with the range $0.90 \leq b \leq 0.95$ obtained by Matthews [13] when a significant inelasticity in pion interactions is included. Nevertheless, statements about composition cannot be made yet from these preliminary experimental results obtained with the UMD engineering array due to the still large statistical uncertainties and the ongoing study of systematic uncertainties. 


\section{Summary}

An improved reconstruction method for the underground muon detector of AMIGA was developed with the aim of removing the overcount due to corner-clipping muons which, with the former reconstruction method, could be as high as $\sim 20 \%$ for zenith angles of $45^{\circ}$. The new method, based on a parametrization of the bias obtained from shower and detector simulations, uses the SD reconstructed angles $\theta$ and $\varphi$ and works well for all distances, primaries, models and energies. The improved method was applied to experimental data acquired by the UMD engineering array, and preliminary results of the muon content estimator $\rho_{450}$ were presented as a function of energy. The logarithmic gain of muons with growing energy was found to be $0.90 \pm 0.03$, which is in good agreement with models in which a significant inelasticity in pion interactions is included. Despite the fact that conclusions about composition cannot yet be drawn due to high statistical uncertainties and not yet accurately estimated systematics, the potentiality of the direct measurement of the muon component by the UMD of AMIGA is shown.

\section{References}

[1] A. Aab et al., The Pierre Auger Collaboration, Nucl. Instrum. Methods A 798 (2015) 172.

[2] A. Aab et al., The Pierre Auger Collaboration, JINST 11 (2016) P02012.

[3] A. Aab et al., The Pierre Auger Collaboration, JINST 12 (2017) P03002.

[4] I. C. Mariş, The AMIGA infill detector of the Pierre Auger Observatory: Performance and first data, in proceedings of 32nd International Cosmic Ray Conference, Beijing, China, vol. 1, p. 9, 2011 [arXiv:1107.4809].

[5] Hamamatsu, Photomultiplier tube assembly H8804, 2012. See http://www.hamamatsu.com/resources/pdf/etd/H8804_TPMH1333E.pdf.

[6] B. Wundheiler, The AMIGA muon counters of the Pierre Auger Observatory: Performance and first data, in proceedings of 32nd International Cosmic Ray Conference, Beijing, China, vol. 5, p. 9, 2011 [arXiv:1107.4807].

[7] D. Ravignani et al., Astropart. Phys. 82 (2016) 108.

[8] S. Ostapchenko, Phys. Rev. D 83 (2011) 014018.

[9] D. Heck et al., CORSIKA: A Monte Carlo code to simulate extensive air showers, Forschungszentrum Karlsruhe Report FZKA 6019, 1998. See http://bibliothek.fzk.de/zb/berichte/FZKA6019.pdf.

[10] T. Pierog and K. Werner, Nucl. Phys. B 196 (2009) 102.

[11] S. Argirò et al., Nucl. Instrum. Methods A $\mathbf{5 8 0}$ (2007) 1485.

[12] B. Wundheiler, The AMIGA muon counters of the Pierre Auger Observatory: Performance and studies of the lateral distribution function, in proceedings of 34th International Cosmic Ray Conference, The Hague, The Netherlands, POS ( ICRC2015) 324 (2015).

[13] J. Matthews, Astropart. Phys. 22 (2005) 387. 